\title{
Adaptive Time-delay Estimated Sliding Mode Control for a Bias Momentum Satellite with Two Reaction Wheels*
}

\author{
Hirohisa KoJIMA $^{1{ }^{\dagger}}$ and Pavel M. TRIVAILO ${ }^{2)}$ \\ ${ }^{1)}$ Department of Aeronautics and Astronautics, Tokyo Metropolitan University, Hino, Tokyo 191-0065, Japan \\ ${ }^{2}$ School of Engineering, RMIT University, Bundoora, Victoria 3083, Australia
}

\begin{abstract}
An adaptive sliding mode control to stabilize the attitude of a bias momentum satellite with a time delay via two wheels is proposed. Stabilizing the attitude of a rigid body via two control torques is an under-actuated control problem, and belongs to the class of systems that are controllable but cannot be asymptotically stabilized via continuous state feedback because Brockett's necessary condition is not satisfied. The adaptive method combined with the sliding mode control estimates the time delay contained in the system by sensing the difference between the attitude predicted and the measured one at each sampling time and compensates for the time delay by predicting the current state using the past measured attitude and angular velocity. Provided that external disturbances and modeling uncertainties in the satellite moments of inertia are absent, the validity of the proposed adaptive time delay estimated sliding mode control for attitude control of a bias momentum satellite is verified through numerical simulations.
\end{abstract}

Key Words: Bias Momentum Satellite, Two-wheel Control, Time-delay Estimation, Sliding Mode Control

\section{Nomenclature}

$\boldsymbol{B}$ : matrix determined from $\boldsymbol{p}$

$\boldsymbol{c}$ : unit vector representing the LOS to the image center, $\boldsymbol{c}=[0,0,1]^{T}$

cmos $_{x}$ : $x$ position of the scaled target along the $x$-axis of the camera

$\operatorname{cmos}_{y}: y$ position of the scaled target along the $y$-axis of the camera

$\boldsymbol{H}_{i}$ : angular momentum of the satellite represented in the inertial frame

$\boldsymbol{I}_{n \times n}: n \times n$ unit matrix

$\boldsymbol{J}$ : moment of inertia tensor of the satellite, $\boldsymbol{J}=\operatorname{diag}\left(J_{1}, J_{2}, J_{3}\right), \mathrm{kgm}^{2}$

$\boldsymbol{J}_{w 1}, \boldsymbol{J}_{w 3}$ : moment of inertia tensor of wheels, $\mathrm{kgm}^{2}$

$j$ : horizon for the adaptive law of time-delay estimation

$\boldsymbol{K}$ : control gain matrix for sliding mode control

$k_{n}, k_{w}$ : wheel speed control gains

$\ell$ : angular momentum vector for the wheels in the body-fixed frame, $\ell=\left[\ell_{1}, 0, \ell_{3}\right]^{T}$

$\boldsymbol{m}$ : unit vector representing the line of sight to the target

$n_{w 1}, n_{w 3}$ : control torques applied to Wheels 1 and $3, \mathrm{Nm}$

$\boldsymbol{p}$ : Rodrigues parameter, $\boldsymbol{p}=\left[p_{1}, p_{2}, p_{3}\right]^{T}$

$\boldsymbol{p}^{\times}$: skew symmetric matrix derived from the vector, $\boldsymbol{p}$

$S: 3 \times 3$ matrix used to design the sliding surface

$(\boldsymbol{S B})^{\#}$ : pseudo inverse of $\boldsymbol{S B}$

$T$ : sampling period for taking the target image, $\mathrm{s}$

$t$ : time, $\mathrm{s}$

(C) 2019 The Japan Society for Aeronautical and Space Sciences

*Received 21 August 2018; final revision received 25 January 2019; accepted for publication 8 February 2019.

†Corresponding author, hkojima@tmu.ac.jp
$\boldsymbol{U}$ : control input

$z_{1}, z_{3}$ : rotational axes of Wheels 1 and 3

$\gamma$ : gain of adaptive law for time-delay estimation

$\delta$ : dead band parameter for adaptive law

$\dot{\theta}_{w 1}, \dot{\theta}_{w 3}$ : rotational speeds of Wheels 1 and $3, \mathrm{rad} / \mathrm{s}$

$\hat{\theta}_{w 1}, \hat{\theta}_{w 3}$ : angular velocity commands for wheels (Wheels 1 and 3), $\mathrm{rad} / \mathrm{s}$

$\dot{\theta}_{w 3 i}$ : initial angular velocity of momentum wheel (Wheel 3), $\mathrm{rad} / \mathrm{s}$

$\tau$ : time delay, $\mathrm{s}$

$\hat{\tau}_{k}$ : estimated time delay at sampling time $t_{k}$

$\sigma$ : sliding manifold

$\Phi_{i}^{b}$ : direct cosine matrix from inertial frame to satellite body-fixed frame

$\boldsymbol{\omega}$ : angular velocity of satellite, $\boldsymbol{\omega}=\left[\omega_{1}, \omega_{2}, \omega_{3}\right]^{T}$

\section{Introduction}

It is impossible to repair or replace malfunctioning attitude control torquers after a satellite is launched. It is, however, desirable to maintain the attitude control ability of the satellite in order to complete the mission and extend the lifespan of the satellite, even if one of the three-axis control torquers, such as a reaction wheel, fails. For this reason, the problem of stabilizing a rigid spacecraft using fewer than three controls has been investigated in a number of studies. Stabilization of an asymmetrical rigid body via two control torques is classified into two stabilization problems: stabilization of the angular velocities and stabilization of the attitude. The problem of stabilizing the angular velocities using fewer than three control torques has been investigated in numerous studies. ${ }^{1-10)}$ The attitude of an asymmetrical rigid body with only two controls belongs to the class of systems that are controllable but cannot be asymptotically stabilized via continuous 
state feedback. This is because Brockett's necessary condition for the existence of a continuously differential control law ${ }^{2)}$ is not satisfied for this system. ${ }^{\ddagger}$ However, this system can be stabilized by other nonlinear control schemes. Such schemes can be categorized as discontinuous control schemes ${ }^{11,12)}$ or time-varying control schemes. ${ }^{13-15)}$ For the special case in which an axisymmetrical spacecraft has an angular velocity of zero around the axisymmetrical axis, smooth state feedback control can still stabilize the attitude of the spacecraft. ${ }^{16,17)}$ Some studies have considered the use of gas-jet thrusters, reaction wheels, or control moment gyros to generate two torques for pointing control. Tsiotras and Longuski described spin-axis stabilization of a symmetrical spacecraft with two control torques generated by gas-jet thrusters. ${ }^{18)}$ Zavoli et al. reported single-axis pointing of an underactuated spacecraft with two reaction wheels. ${ }^{19)}$ Shen and Tsiotras investigated time-optimal control of an axisymmetrical rigid body using two wheels. ${ }^{20)}$ Endo and Ueno studied minimum-energy maneuvering of a satellite using two wheels ${ }^{21)}$ using the coordinate system proposed by Tsiotras and Longuski. ${ }^{22)}$ Tsuchiya et al. proposed a Lyapunov-based discontinuous controller using two wheels. ${ }^{23)}$ Shimoda et al. studied attitude control with trajectory planning. ${ }^{24,25)}$ Yamada and Yoshikawa presented a control method using listing parameters for the two-wheel problem. ${ }^{26,27)}$ Kasai et al. presented a feedforward attitude maneuver method using two single-gimbal control moment gyros. ${ }^{28)}$ Kojima et al. described a line-of-sight attitude maneuvering control using two single-gimbal control moment gyros. ${ }^{29)}$ Terui et al. reported the attitude maneuvering of a bias momentum satellite using two wheels and verified their control scheme using $\mu$ LabSat in orbit. ${ }^{30)}$ As reported by Terui et al., ${ }^{30)}$ the control system of a satellite based on image processing contains a time delay, which deteriorates the control performance, and a long time delay could make the system unstable.

A number of methods have been proposed to estimate/ identify the time delay; for example, as reported by Zheng et al. $^{31)}$ However, to the best of our knowledge, a method by which to compensate for the time delay of the attitude stabilization of a bias momentum satellite subject to the twowheel control problem has not yet been proposed. In this paper, an adaptive sliding mode control to stabilize the attitude of a bias momentum satellite considering time delay is proposed. The bias momentum satellite model in this study is basically the same as that for the $\mu$-LabSat, but is slightly simplified. The objectives of the adaptive law are to estimate the time delay by sensing the difference between the attitude predicted using the angular velocity measured and the attitude measured at the sampling time and to compensate for the delay by predicting the current state based on the measured state.

Numerical simulation will show that attitude stabilization can be achieved by the proposed adaptive time-delay esti-

\footnotetext{
†Theorem (Brockett): The linearized system should have no uncontrollable mode associated with eigenvalues whose real parts are positive, and $f(\boldsymbol{x}, \boldsymbol{u})=\boldsymbol{y}$ is solvable with respect to $(\boldsymbol{x}, \boldsymbol{u})$ for all sufficiently small $\|\boldsymbol{y}\|$.
}

mated sliding mode control (ATDESMC), and that ATDESMC is effective for attitude maneuvering, as compared to the sliding mode control described by Terui et al. ${ }^{30)}$

The remainder of this paper is organized as follows. In Section 2, the equations of motion and problem statement are presented. In Section 3, the sliding mode controller using two wheels is described. In Section 4, an adaptive law to estimate the time delay is addressed. The results of numerical simulations are presented in Section 5 in order to demonstrate the validity of the proposed method. Finally, the conclusion of this study is presented in Section 6.

\section{Equations of Motion and Problem Statement}

\subsection{Equations of motion}

It is assumed that the principal axes of a satellite are coincident with its body frame coordinates and that the satellite has a bias moment wheel, Wheel 3 , located along the $z$-axis of the body-fixed frame. Although the axis of Wheel 1 was inclined $30 \mathrm{deg}$ around the $z$-axis of the body frame in the $\mu$-LabSat, in this study, Wheel 1 is assumed to be located along the $x$-axis of the body-fixed frame, as shown in Fig. 1. In this case, the equations of motion are given by

$$
\left[\begin{array}{c}
J_{1} \dot{\omega}_{1} \\
J_{2} \dot{\omega}_{2} \\
J_{3} \dot{\omega}_{3}
\end{array}\right]=\left[\begin{array}{c}
\left(J_{2}-J_{3}\right) \omega_{2} \omega_{3} \\
\left(J_{3}-J_{1}\right) \omega_{3} \omega_{1} \\
\left(J_{1}-J_{2}\right) \omega_{1} \omega_{2}
\end{array}\right]-\left[\begin{array}{c}
n_{w 1}+\omega_{2} \ell_{3} \\
\omega_{3} \ell_{1}-\omega_{1} \ell_{3} \\
n_{w 3}-\omega_{2} \ell_{1}
\end{array}\right] .
$$

Note that no control torque is applied to the $y$-axis. The equations of motion for the wheels are given by

$$
\left[\begin{array}{l}
\dot{\ell}_{1} \\
\dot{\ell}_{3}
\end{array}\right]=\left[\begin{array}{c}
J_{w 1}(1,1) \ddot{\theta}_{w 1} \\
J_{w 3}(3,3) \ddot{\theta}_{w 3}
\end{array}\right]=\left[\begin{array}{c}
n_{w 1} \\
n_{w 3}
\end{array}\right] .
$$

\subsection{Attitude kinematics}

Figure 2 shows the coordinate system for the camera. Although the line of sight of the camera was coincident with the direction opposite to the $z$-axis in the study by Terui et al., ${ }^{30)}$ for simplicity, it is assumed in this study that the line of sight of the camera is coincident with the $z$-axis of the body frame. Under this assumption, the unit vector corresponding to the image center in the body-fixed coordinate system, $\boldsymbol{c}$, is given as $\boldsymbol{c}=[0,0,1]^{T}$. The Rodrigues parameter, $\boldsymbol{p}$, is used to represent the attitude of the spacecraft rela-

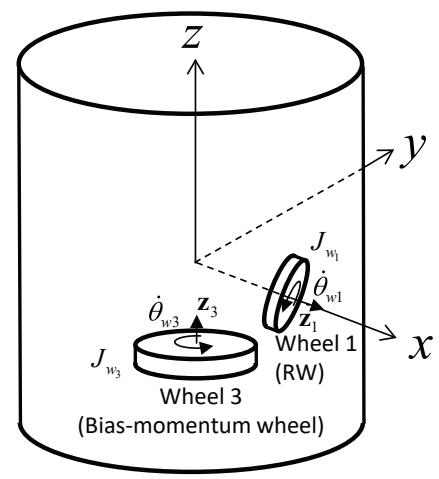

Fig. 1. Bias-momentum satellite model. 


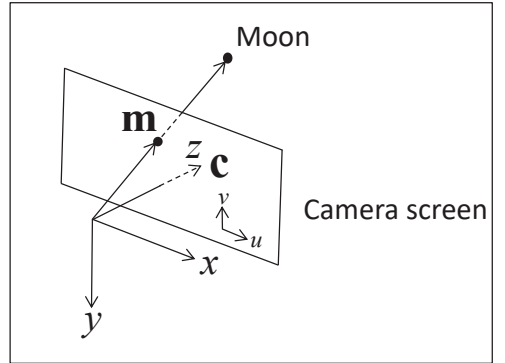

Fig. 2. Camera coordinate system.

tive to the direction of the Moon, but not to the inertial frame. In this case, $\boldsymbol{p}$ can be expressed using vectors $\boldsymbol{m}$ and $\boldsymbol{c}$ as

$$
p= \begin{cases}\frac{m \times c}{\|m \times c\| \sqrt{\frac{1-m \cdot c}{1+m \cdot c}}} & \|m \times c\| \neq 0 \\ 0 & \|m \times c\|=0 .\end{cases}
$$

Note that in the above-described coordinate system for the camera, $p_{3}$ is always zero.

The time derivative of $\boldsymbol{p}$ is given by

$$
\dot{\boldsymbol{p}}=\frac{1}{2}\left(\boldsymbol{I}_{3 \times 3}+\boldsymbol{p}^{\times}+\boldsymbol{p} \boldsymbol{p}^{T}\right) \omega .
$$

If the attitude state vector sampled at time $t_{k}$ includes a time delay $\tau \in[0, T)$, then the vector can be represented as

$$
\overline{\boldsymbol{p}}(t)=\boldsymbol{p}\left(t_{k}-\tau\right), \quad t_{k} \leq t<t_{k+1}, \quad t_{k}:=k T .
$$

Note that the time derivative of $\boldsymbol{p}$ given by Eq. (4) was not integrated to obtain the current value of $\boldsymbol{p}$ in the study by Terui et al. ${ }^{30)}$

\subsection{Problem statement}

The problem treated herein is to derive an ATDESMC method that can stabilize the attitude of a bias momentum satellite with a time delay in the attitude sensing system based on image processing to the target attitude via two wheels such that the target image is stabilized at the center of the image screen. In other words, the target Rodrigues parameter is a zero vector.

\section{Sliding Mode Control via Two Wheels}

In this section, a sliding mode controller for a bias-momentum satellite is derived by referring to Terui et al., ${ }^{30)}$ with several modifications to the equations of their study.

\subsection{Relationship between attitude kinematics and an- gular momentum of two wheels}

It is assumed that no external torques disturb the attitude motion of the satellite. In this case, the law of conservation of angular momentum is satisfied.

$$
\boldsymbol{J} \boldsymbol{\omega}+\boldsymbol{J}_{w 1}\left(\boldsymbol{\omega}+\boldsymbol{z}_{1} \dot{\theta}_{w 1}\right)+\boldsymbol{J}_{w 3}\left(\boldsymbol{\omega}+\boldsymbol{z}_{3} \dot{\theta}_{w 3}\right)=\Phi_{i}^{b} \boldsymbol{H}_{i}
$$

From Eq. (6), the angular velocity of the satellite is given as

$$
\begin{aligned}
\boldsymbol{\omega}= & -\left(\boldsymbol{J}+\boldsymbol{J}_{w 1}+\boldsymbol{J}_{w 3}\right)^{-1} \boldsymbol{J}_{w 1} z_{1} \dot{\theta}_{w 1} \\
& -\left(\boldsymbol{J}+\boldsymbol{J}_{w 1}+\boldsymbol{J}_{w 3}\right)^{-1} \boldsymbol{J}_{w 3} z_{3} \dot{\theta}_{w 3} \\
& +\left(\boldsymbol{J}+\boldsymbol{J}_{w 1}+\boldsymbol{J}_{w 3}\right)^{-1} \Phi_{i}^{b} \boldsymbol{H}_{i} .
\end{aligned}
$$

Furthermore, assuming that the satellite moments of inertia are much greater than that of the wheels, that is, $\boldsymbol{J}+\boldsymbol{J}_{w 1}+\boldsymbol{J}_{w 3} \approx \boldsymbol{J}$, we have

$$
\boldsymbol{\omega}=-\boldsymbol{z}_{1} v_{1}-\boldsymbol{z}_{3} v_{3}+\boldsymbol{H}_{0}
$$

where

$$
\begin{aligned}
v_{1} & =J_{1}^{-1} J_{w 1}(1,1) \dot{\theta}_{w 1}, \\
v_{3} & =J_{3}^{-1} J_{w 3}(3,3) \dot{\theta}_{w 3}, \\
\boldsymbol{H}_{0} & =J^{-1} \Phi_{i}^{b} \boldsymbol{H}_{i} .
\end{aligned}
$$

Substituting Eq. (8) into Eq. (4) yields

$$
\begin{aligned}
\dot{\boldsymbol{p}}= & -\frac{1}{2}\left(z_{1} v_{1}+z_{3} v_{3}\right)-\frac{1}{2}\left(p_{1} v_{1}+p_{3} v_{3}\right) \boldsymbol{p} \\
& -\frac{1}{2} \boldsymbol{p}^{\times}\left(z_{1} v_{1}+z_{3} v_{3}\right)+\boldsymbol{h}
\end{aligned}
$$

where

$$
\boldsymbol{h}=\frac{1}{2}\left(\boldsymbol{I}_{3 \times 3}+\boldsymbol{p}^{\times}+\boldsymbol{p p}^{T}\right) \boldsymbol{H}_{0}=\left[h_{1}, h_{2}, h_{3}\right]^{T} .
$$

Considering the first and third elements on both sides of Eq. (12) and solving the equation with respect to $v_{1}$ and $v_{3}$ yields

$\left[\begin{array}{l}v_{1} \\ v_{3}\end{array}\right]=\frac{2}{1+\boldsymbol{p}^{T} \boldsymbol{p}}\left[\begin{array}{cc}-\left(1+p_{3}^{2}\right) & p_{1} p_{3}+p_{2} \\ p_{1} p_{3}-p_{2} & -\left(1+p_{1}^{2}\right)\end{array}\right]\left[\begin{array}{c}\dot{p}_{1}-h_{1} \\ \dot{p}_{3}-h_{3}\end{array}\right]$.

Substituting the above equation into the second element of Eq. (12), the following equation can be obtained:

$$
\left[\begin{array}{c}
\dot{p}_{1}-h_{1} \\
\dot{p}_{2}-h_{2} \\
\dot{p}_{3}-h_{3}
\end{array}\right]=\left[\begin{array}{cc}
1 & 0 \\
p_{3} & -p_{1} \\
0 & 1
\end{array}\right]\left[\begin{array}{l}
\dot{p}_{1}-h_{1} \\
\dot{p}_{3}-h_{3}
\end{array}\right]=\boldsymbol{B} \boldsymbol{U} .
$$

Note that if $p_{1}$ and $p_{3}$ are non-zero, then $p_{2}$ can be alternated by the attitude change rate around the $x$ - and $z$-axes, that is, the attitude of the bias momentum satellite can be controlled via only two wheels. Moreover, $p_{3}$ is always zero under the coordinate system defined herein. This implies that $p_{1}$ should not be zero in order to control $p_{2}$. On the other hand, if the attitude change rate around the $z$-axis $\left(\dot{p}_{3}-h_{3}\right)$ is zero, then $p_{1}$ can be changed without changing $p_{2}$.

\subsection{Sliding mode controller design}

The sliding manifold $\sigma$ is defined as

$$
\sigma=S p+\dot{p}
$$

where $S$ is a switching surface matrix for the sliding mode controller and must be chosen such that $\boldsymbol{p}$ asymptotically converges to zero when the state lies on the sliding manifold. A Lyapunov function candidate is chosen as 


$$
V=\frac{1}{2} \sigma^{T} \sigma
$$

By taking the time derivative of the above function and recalling Eqs. (15) and (16), we have

$$
\dot{V}=\boldsymbol{\sigma}^{T} \dot{\boldsymbol{\sigma}}=\boldsymbol{\sigma}^{T}(\boldsymbol{S B U}+\boldsymbol{S h}+\ddot{\boldsymbol{p}}) .
$$

Note that $\ddot{\boldsymbol{p}}$, which must be included, was not included in the time derivative of the sliding manifold in Ref. 30).

Let us assume that there is an upper bounded value for the absolute value of $\boldsymbol{S h}+\ddot{\boldsymbol{p}}$, as follows:

$$
\begin{aligned}
& \boldsymbol{S h}+\ddot{\boldsymbol{p}}=\boldsymbol{\Psi}=\left[\begin{array}{lll}
\Psi_{1} & \Psi_{2} & \Psi_{3}
\end{array}\right]^{T}, \\
& \left|\Psi_{i}\right| \leq \Psi_{i \max } \quad(i=1,2,3) .
\end{aligned}
$$

This assumption means that the control torque acting on the satellite is restricted within some limited value. Let us design a sliding mode controller as

$$
\boldsymbol{U}=-(\boldsymbol{S B})^{\#} \boldsymbol{K} \operatorname{sgn}(\boldsymbol{\sigma})
$$

where

$$
\begin{aligned}
\boldsymbol{K} & =\operatorname{diag}\left(K_{1}, K_{2}, K_{3}\right) \\
& =\operatorname{diag}\left(k_{1}+\Psi_{1 \max }, k_{2}+\Psi_{2 \max }, k_{3}+\Psi_{3 \max }\right) .
\end{aligned}
$$

Substituting Eqs. (20) and (21) into Eq. (18), we have

$$
\begin{aligned}
\dot{V} & =\boldsymbol{\sigma}^{T}\left\{-(\boldsymbol{S B})(\boldsymbol{S B})^{\#} \boldsymbol{K} \operatorname{sgn}(\boldsymbol{\sigma})+\boldsymbol{S h}+\ddot{\boldsymbol{p}}\right\} \\
& =-\sum_{i=1}^{3} K_{i}\left|\sigma_{i}\right|+\boldsymbol{\sigma}^{T}(\boldsymbol{S} \boldsymbol{h}+\ddot{\boldsymbol{p}}) \\
& \leq-\sum_{i=1}^{3}\left(k_{i}+\Psi_{i \max }\right)\left|\sigma_{i}\right|+\sum_{i=1}^{3} \Psi_{i \max }\left|\sigma_{i}\right| \\
& =-\sum_{i=1}^{3} k_{i}\left|\sigma_{i}\right| \leq 0 .
\end{aligned}
$$

From the above inequality, the time derivative of the Lyapunov function candidate is negative for a non-zero sliding manifold value. This implies that the Lyapunov function converges to zero, that is, the existence of the reaching mode is proven.

When the sliding manifold is zero, by multiplying $\boldsymbol{p}^{T}$ from the left-hand side of Eq. (16), we have

$$
\boldsymbol{p}^{T} \dot{\boldsymbol{p}}=-\boldsymbol{p}^{T} \boldsymbol{S p} \text {. }
$$

The left-hand side of Eq. (23) is the time derivative of $V_{2}=\boldsymbol{p}^{T} \boldsymbol{p} / 2$. If a positive definite matrix is chosen for $S$, and the minimum eigenvalue of $S$ is given by $\lambda_{\min }$, and then

$\dot{V}_{2}=\frac{d}{d t}\left(\frac{1}{2} \boldsymbol{p}^{T} \boldsymbol{p}\right) \leq-\lambda_{\min }\|\boldsymbol{p}\|^{2}=-2 \lambda_{\min } V_{2}<0$ for $\boldsymbol{p} \neq 0$.

This leads to

$$
V_{2}(t) \leq V_{2}\left(t^{\prime}\right) e^{-2 \lambda_{\min }\left(t-t^{\prime}\right)}
$$

where $t^{\prime}$ is the time when the state reaches the sliding manifold. Consequently, the sliding mode is achieved, and the sat- ellite attitude will converge to the target attitude as time increases after the state reaches the sliding manifold.

Recalling that $p_{3}$ is always equal to zero, and replacing the sign function in Eq. (20) with a smooth function in order to avoid chattering, substituting Eq. (20) into Eq. (14) and solving with respect to the wheel angular velocity commands using Eqs. (9) and (10), we have

$$
\begin{aligned}
{\left[\begin{array}{c}
\dot{\hat{\theta}}_{w 1} \\
\dot{\hat{\theta}}_{w 3}
\end{array}\right]=} & \frac{-2}{1+p_{1}^{2}+p_{2}^{2}}\left[\begin{array}{cc}
J_{1} J_{w 1}(1,1)^{-1} & 0 \\
0 & J_{3} J_{w 3}(3,3)^{-1}
\end{array}\right] \\
& \times\left[\begin{array}{cc}
-1 & p_{2} \\
-p_{2} & -\left(1+p_{1}^{2}\right)
\end{array}\right](\mathbf{S B})^{\#} \boldsymbol{K} \frac{\sigma}{|\sigma|+\varepsilon} \cdot
\end{aligned}
$$

In Ref. 30), the following speed control method is used to implement the controller for driving the wheels.

$$
\begin{aligned}
& n_{w 1}=k_{n} k_{w} J_{w 1}(1,1)\left(\dot{\hat{\theta}}_{w 1}-\dot{\theta}_{w 1}\right) \\
& n_{w 3}=k_{n} k_{w} J_{w 3}(3,3)\left(\dot{\theta}_{w 3 i}+\dot{\hat{\theta}}_{w 3}-\dot{\theta}_{w 3}\right)
\end{aligned}
$$

Although the gain for the torque command $k_{n}$ was scaled in Ref. 30) as

$$
k_{n}=k_{0}+\sqrt{\operatorname{cmos}_{x}^{2}+\operatorname{cmos}_{y}^{2}},
$$

for simplicity, here $k_{n}$ is set to a constant value.

As described in Ref. 30), the sliding mode control method presented here basically exhibits two stages: 1) rotation around the $z$-axis to stabilize $p_{2}$ to zero using Wheel 3 , and 2) rotation around the $x$-axis to stabilize $p_{1}$ to zero using Wheel 1. In addition, the bias momentum satellite has a tendency to shift its bias momentum axis in a clockwise direction in the camera view due to nutation. As a result of this clockwise nutation, the trajectory of the target image on the camera screen likely results in a counter-clockwise motion.

\section{Adaptive Law for Estimating Time Delay}

Based on Ref. 30), the bias momentum satellite assumed in this study is the $\mu$-LabSat, which took a target image approximately every $2 \mathrm{~s}$ to track the direction to the target. In this situation, by applying the time that has passed after taking the target image, the current attitude can be estimated by integrating the equations of motion forward in time from the measured attitude and angular velocity sensed by the inertial reference unit. Note, however, the attitude signal may still contain another time delay for other reasons, such as image processing time. The adaptive law described here is intended to estimate and compensate for such unexpected time delays, provided that modeling uncertainties for the satellite are absent. The adaptive law proposed here is a discrete Newtonbased method to estimate the time delay. The concept is based on a time-delay continuous estimation method presented by Zheng et al., ${ }^{31)}$ but by taking into consideration that the sampling period of taking target images in the $\mu$ - 
LabSat was $2.0 \mathrm{~s}$, a continuous adaptive law is modified to a discrete adaptive law. The error between $\boldsymbol{p}$ estimated based on the estimated time delay and $\boldsymbol{p}$ measured is defined as

$$
\boldsymbol{\Delta}\left(t_{k}, \hat{\tau}_{k}\right)=\hat{\boldsymbol{p}}\left(t_{k}, \hat{\tau}_{k}\right)-\overline{\boldsymbol{p}}\left(t_{k}\right) .
$$

A non-negative performance index with respect to the timedelay estimation error is selected as

$$
Q\left(t_{k}, \hat{\tau}_{k}\right)=\sum_{i=k-j+1}^{k} \Delta^{2}\left(t_{i}, \hat{\tau}_{k}\right) \geq 0 .
$$

The discrete time derivative of the performance index is given as

$$
\Delta Q\left(t_{k}, \hat{\tau}_{k}\right)=\frac{\partial Q\left(t_{k}, \hat{\tau}_{k}\right)}{\partial \hat{\tau}_{k}} \Delta \hat{\tau}_{k}
$$

In this study, it is assumed that

$$
\frac{\partial Q\left(t_{k}, \hat{\tau}_{k}\right)}{\partial \hat{\tau}_{k}} \neq 0 .
$$

The discrete adaptive law to update the estimated time delay is designed as

$$
\begin{aligned}
\Delta \hat{\tau}_{k} & =-\frac{\gamma Q\left(t_{k}, \hat{\tau}_{k}\right)}{\frac{\partial Q\left(t_{k}, \hat{\tau}_{k}\right)}{\partial \hat{\tau}_{k}}}, \\
\hat{\tau}_{k+1} & =\hat{\tau}_{k}+\Delta \hat{\tau}_{k}
\end{aligned}
$$

where $\gamma$ is a discount factor of the performance index, $Q\left(t_{k}, \hat{\tau}_{k}\right)$, and thus should be chosen as $0<\gamma<1$. Substituting Eq. (34) into Eq. (32) yields

$$
\Delta Q\left(t_{k}, \hat{\tau}_{k}\right)=-\gamma Q\left(t_{k}, \hat{\tau}_{k}\right)
$$

A schematic image of the adaptive law is shown in Fig. 3, and a flowchart is shown in Fig. 4. In the above adaptive law, Eq. (33) is assumed. However, the value of the left-hand side of Eq. (33) might become too small, and a very large updated value for the time delay will be derived. In such a case, the estimated time delay will lose physical appropriateness. In order to avoid such a situation, the following ad-hoc procedure is implemented in this study:

$$
\Delta \hat{\tau}_{k}= \begin{cases}-\frac{\gamma Q\left(t_{k}, \hat{\tau}_{k}\right)}{\frac{\partial Q\left(t_{k}, \hat{\tau}_{k}\right)}{\partial \hat{\tau}_{k}}} & : \frac{\partial Q\left(t_{k}, \hat{\tau}_{k}\right)}{\partial \hat{\tau}_{k}}<-\delta \\ 0 & : \text { otherwise. }\end{cases}
$$

Note that, as a result of the above updating procedure, the estimated time delay increases.

After updating the estimated time delay, the Rodrigues parameters at the sampling time and current time are predicted by integrating the equation of motion forward in time with the updated time delay from the output attitude signal to the sampling time and current time, respectively, as follows:

$$
\begin{aligned}
\hat{\boldsymbol{p}}_{i+1}\left(t_{i}\right) & =\boldsymbol{p}_{i}\left(t_{i}\right)+\int_{t_{i}-\hat{\tau}_{k}}^{t_{i+1}-\hat{\tau}_{k}} \dot{\hat{\boldsymbol{p}}}(\hat{\boldsymbol{p}}(\xi), \omega(\xi)) d \xi \\
(i & =k-j, \cdots, k-1),
\end{aligned}
$$

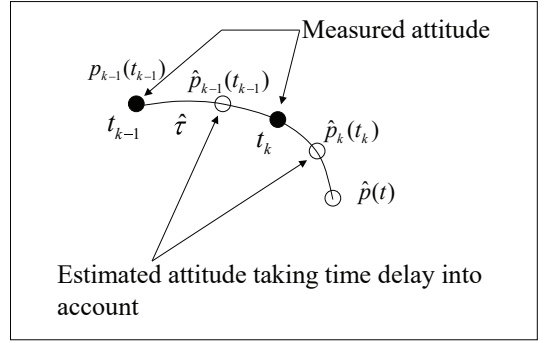

Fig. 3. Schematic representation of the adaptive time delay estimation law.

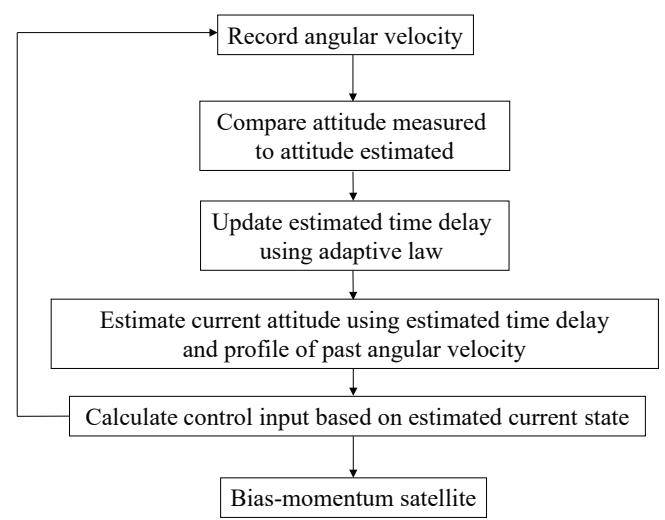

Fig. 4. Flowchart of adaptive time-delay estimation.

$$
\hat{\boldsymbol{p}}(t)=\boldsymbol{p}_{k}\left(t_{k}\right)+\int_{t_{k}-\hat{\tau}_{k}}^{t} \dot{\hat{\boldsymbol{p}}}(\hat{\boldsymbol{p}}(\xi), \omega(\xi)) d \xi
$$

The sliding manifold in Eq. (16) is replaced with the manifold resulting from $\boldsymbol{p}$ predicted and its time derivative, as follows:

$$
\sigma=S \hat{p}+\dot{\hat{p}} .
$$

If the estimated time delay is correct, then the current attitude is also correctly estimated and the performance of the controller after canceling the time delay is the same as that of the system without the time delay. Even if the estimated time delay is not always correct, it can be expected that the performance of the controller will be improved as result of the above prediction scheme.

\section{Numerical Simulations}

\subsection{Numerical conditions}

The parameters, which are approximately the same as those in Ref. 30), except for the sliding surface matrix $S$ and $\varepsilon$, are listed in Tables 1 and 2. For the case in which the initial target's position is located in the 2nd (or 4th) quadrant of the camera screen, the target image moves through the 3rd (or 1st) quadrant of the camera screen until convergence of $p_{2}$ (or $u$ ) to zero without changing the distance between the target image and the origin of the camera screen if the 1st control stage is simply carried out. These trajectories are undesirable because the target may leave the screen. In order to avoid such undesirable trajectories, the attitude change rate 
Table 1. Simulation parameters

\begin{tabular}{ll}
\hline \multicolumn{1}{c}{ Parameter } & \multicolumn{1}{c}{ Value $[$ units $]$} \\
\hline$\left(J_{1}, J_{2}, J_{3}\right)$ & $(2.86,2.86,3.15)\left[\mathrm{kgm}^{2}\right]$ \\
$J_{w 1}(1,1), J_{w 3}(3,3)$ & $0.00239,0.00239\left[\mathrm{kgm}^{2}\right]$ \\
$\ell_{0}\left(=J_{w 3}(3,3) \dot{\theta}_{w 3 i}\right)$ & $0.934[\mathrm{Nms}]$ \\
Absolute maximum control torque $n_{\max }$ & $0.023[\mathrm{Nm}]$ \\
\hline Image processing sampling time $T$ & $2.0[\mathrm{~s}]$ \\
Control cycle & $1.0[\mathrm{~s}]$ \\
\hline Size of image & $640 \times 480[\mathrm{pixels}]$ \\
Angle of view & $70 \times 90[\mathrm{deg}]$ \\
\hline Initial attitude $\left(p_{1}, p_{2}, p_{3}\right)$ & Case $1:(-0.08,-0.08,0)$ \\
& Case $2:(-0.08,0.08,0)$ \\
Initial angular velocity $\omega$ & $(0,0,0)[\mathrm{rad} / \mathrm{s}]$ \\
\hline $\boldsymbol{K}$ & diag $(10,25,10)$ \\
$k_{n}, k_{w}, \varepsilon$ & $0.2,0.001,0.1$ \\
$j, \gamma$ & $5,0.8$ \\
$\delta($ ATDESMC $(1))$ & $1.0 \times 10^{-7}$ \\
$\delta($ ATDESMC $(2))$ & $2.0 \times 10^{-5}$ \\
\hline Maximum time delay & $4.0[\mathrm{~s}]$ \\
Initial estimated time delay $\hat{\tau}_{0}$ & $0.0[\mathrm{~s}]$ \\
\hline
\end{tabular}

Table 2. Selection of sliding surface $S$ based on the initial target's position on the camera screen.

\begin{tabular}{cccc}
\hline Quadrant & \multicolumn{3}{c}{$\boldsymbol{S}$} \\
\hline \multirow{3}{*}{ 1st } & {$\left[\begin{array}{ccc}0.0724 & 0 & 0 \\
0 & 0.105 & 0.0125 \\
0 & 0.0125 & 0.0063\end{array}\right]$} \\
2nd & {$\left[\begin{array}{ccc}0.0270 & 0 & 0 \\
0 & 0.105 & -0.0125 \\
0 & -0.0125 & 0.0063\end{array}\right]$} \\
& {$\left[\begin{array}{ccc}0.0724 & 0 & 0 \\
0 & 0.105 & -0.0125 \\
0 & -0.0125 & 0.0063\end{array}\right]$} \\
3rd & {$\left[\begin{array}{ccc}0.0270 & 0 & 0 \\
0 & 0.105 & 0.0125 \\
0 & 0.0125 & 0.0063\end{array}\right]$}
\end{tabular}

around the $x$-axis should be controlled simultaneously, but $p_{1}$ (or $v$ ) should not converge to zero before $p_{2}$ (or $u$ ) converges to zero. As the element of the sliding surface matrix $S$ becomes smaller, the element of the pseudo matrix of $\boldsymbol{S B}$ becomes larger. This implies that $p_{1}$ converges to zero faster as the element $(1,1)$ of $\boldsymbol{S}$ becomes smaller. By taking these points into consideration, the sliding surface matrix $\boldsymbol{S}$ was selected based on the initial position of the target on the camera screen, as listed in Table 2.

Although a non-symmetric matrix was set for $S$ in Ref. 30), a symmetric positive definite matrix was chosen here, and a different element $(1,1)$ of $\boldsymbol{S}$ was chosen as well.

The time delay induced due to image processing, $\tau$, is assumed to be $2.0 \mathrm{~s}$, but is not known in advance. Since the sampling time $T$ is $2 \mathrm{~s}$, the maximum time delay for the attitude measurement is $4.0 \mathrm{~s}$, which is assumed to be constant in this study. The control cycle was $1 \mathrm{~s}$ and was the same as that in the $\mu$-LabSat. External disturbance torques and modeling uncertainties, except for the time delay, are assumed to be absent.

Numerical simulations are carried out to demonstrate the validity of ATDESMC. Two cases are investigated in order to validate the independence of the proposed method from the initial position of the target on the screen. The initial target on the camera screen is in the 1st quadrant for Case 1 and 2nd quadrant for Case 2. Since the results for the cases in which the initial target is in the 3rd and 4th quadrants are basically the same as those for the cases in which the initial target is in the 1st and 2nd quadrants, respectively, these results are omitted in this paper.

In addition, the influence of the parameter for updating the time-delay estimation $\delta$ on the control performance is studied by considering two values of $\delta$ : ATDESMC (1) and ATDESMC (2).

\subsection{Results of numerical simulations}

Figure 5 shows the numerical simulation results of SMC for Case 1, including the time response of the angular velocities, the trajectory of the target on the camera screen, and the time history of the control torques. Figure 6 shows the numerical simulation results of ATDESMC for Case 1, and Fig. 7 shows the time histories of the estimated time delay for two values of $\delta$. Figure 8 compares the trajectories resulting from SMC and ATDESMC for Case 1. Figures 9 and 10 show the numerical simulation results of SMC for Case 2 and the results of ATDESMC for Case 2, respectively. Figure 11 shows the influence of $\delta$ on the time delay estimation. Figure 12 compares the trajectories for Case 2. Note that Figs. 6 and 10 show the results for the case of $\delta=1.0 \times 10^{-7}($ ATDESMC (1).

First, the results for Case 1 are discussed. In Figs. 5(a) and 5 (b), the angular velocities converged to zero and the attitude was successfully controlled to stabilize the target at the origin of the camera screen by SMC, but a large negative overshoot of $\omega_{z}$ and a small negative overshoot of the target's position in $u$ occurred before convergence. The settling time was approximately $150 \mathrm{~s}$ for SMC. In addition, a small oscillating control torque was generated in $n_{w 3}$, and a small smooth torque was generated in $n_{w 1}$ to stabilize the target's position at the origin of the camera screen, as shown in Fig. 5(c).

Similarly, ATDESMC succeeded in stabilizing the angular velocities to zero and stabilized the target at the origin of the camera screen, as shown in Figs. 6(a) and 6(b), respectively. A large negative overshoot occurred in $\omega_{z}$, but its settling time was approximately $130 \mathrm{~s}$ for ATDESMC, which was shorter that for SMC. In addition, a negative overshoot of the target's position in $u$ occurred before convergence for ATDESMC, but its magnitude was less than that for SMC, as shown in Fig. 6(b). As shown in Figs. 5(b) and 6(b), the trajectory of the target on the camera screen consists of two stages: 1) stabilization of $u$ to approximately zero, and 2) stabilization of $v$ to zero. This is because the control strategy consists of two stages to maintain the stabilizability, as explained at the end of Subsection 3.2.

The existence of large limit cycles was reported in Ref. 30). However, such large limit cycles did not occur for either SMC or ATDESMC for Case 1. We implemented the numerical simulation code according to the description in Ref. 30), but did not discretize the measured angular velocity and control torque in this study because discretization was 


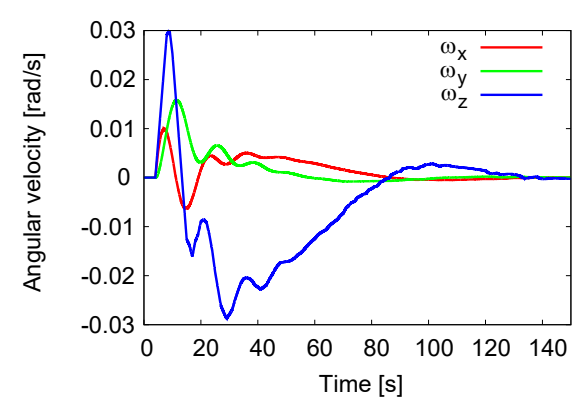

(a)

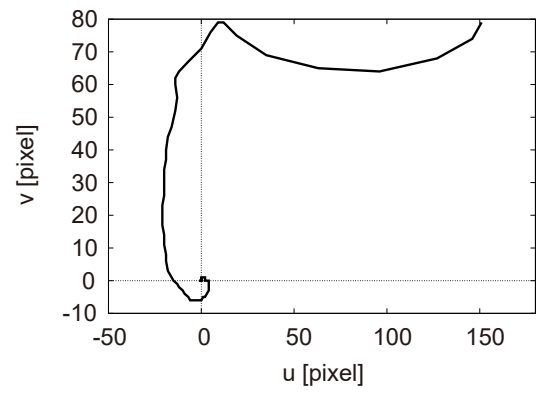

(b)

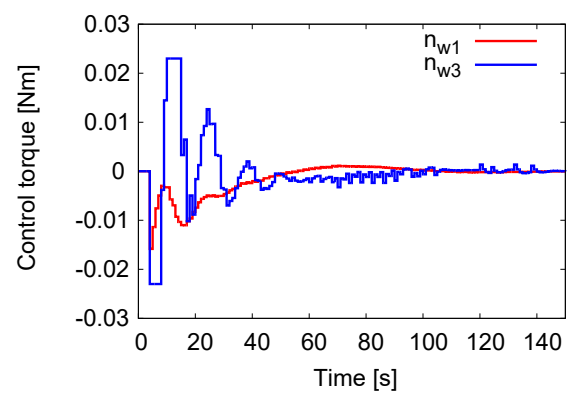

(c)

Fig. 5. Results of SMC for Case 1: (a) angular velocities, (b) trajectory of the target on the screen, and (c) control torques.

not described in detail in Ref. 30). This difference in implementation may be the reason for the presence or absence of limit cycles and their magnitude. The time histories of the estimated time delay for Case 1 are shown in Fig. 7 for two values of $\delta$. For $\delta=1.0 \times 10^{-7}$ (ATDESMC (1)), the estimated time delay was updated until $110 \mathrm{~s}$, which approximately corresponds to the convergence time of the angular velocities. The finally estimated time delay for $\delta=1.0 \times 10^{-7}$ (ATDESMC (1)) was approximately $4 \mathrm{~s}$. Since the sampling time for attitude measurement was $2 \mathrm{~s}$, and the image processing-based attitude determination required a sampling time of $2 \mathrm{~s}$, the maximum time delay was $4 \mathrm{~s}$. Thus, the adaptive time-delay estimation law presented in this study appropriately estimated the time delay for $\delta=1.0 \times 10^{-7}$ in Case 1 . In contrast, for $\delta=2.0 \times 10^{-5}$ (ATDESMC (2)), the estimated time delay was updated until $30 \mathrm{~s}$ and converged to approximately $3 \mathrm{~s}$, which was slightly shorter than the maximum time delay of $4 \mathrm{~s}$. When $\delta$ is set to be larger, the dead band for updating the estimated time delay becomes larger. Thus, the estimated time delay for ATDESMC (2) was less likely to be updated than ATDESMC (1). As a result of time delay estimation, for ATDESMC, the trajectory on the camera screen was more efficiently stabilized to the origin than

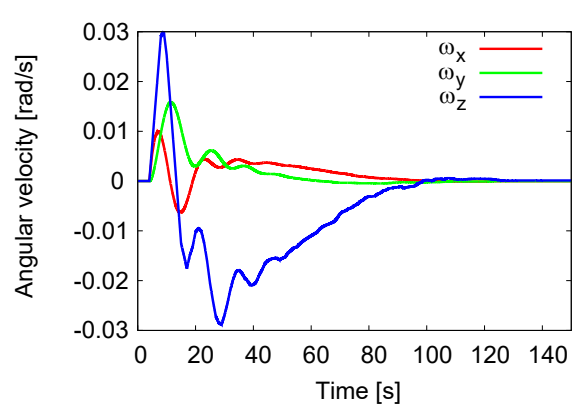

(a)

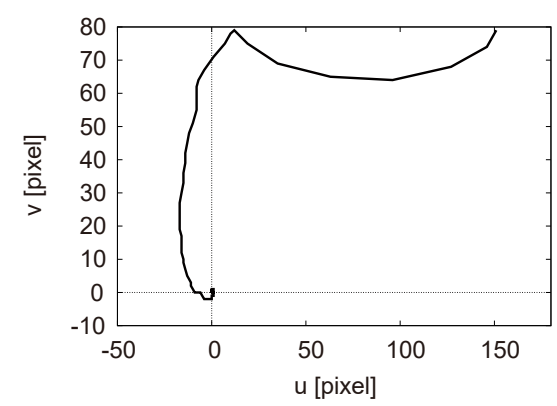

(b)

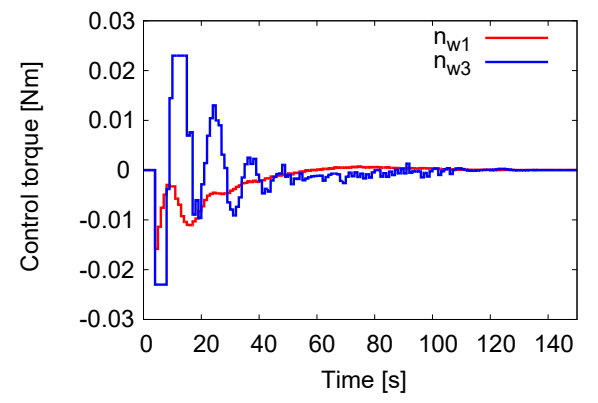

(c)

Fig. 6. Results for ATDESMC for Case 1: (a) angular velocities, (b) trajectory of the target on the screen, (c) control torques.

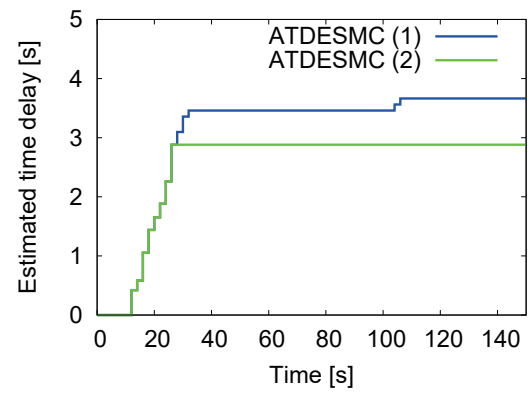

Fig. 7. Time histories of the time-delay estimation for Case 2.

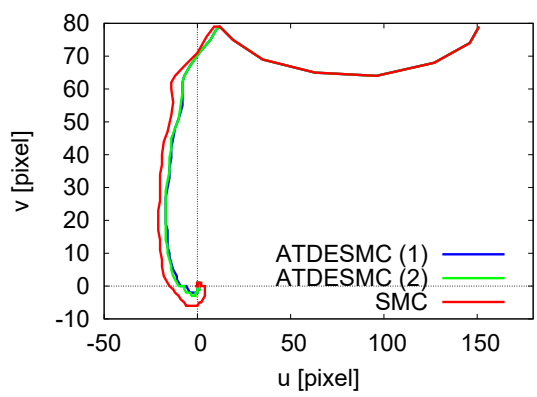

Fig. 8. Comparison of trajectories for Case 1. 


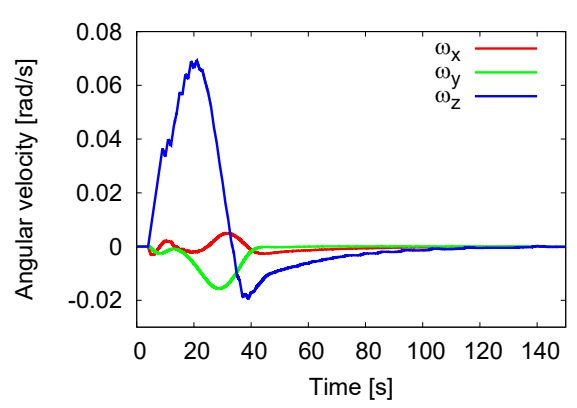

(a)

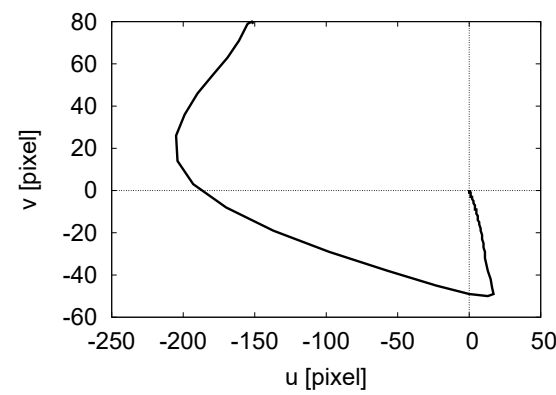

(b)

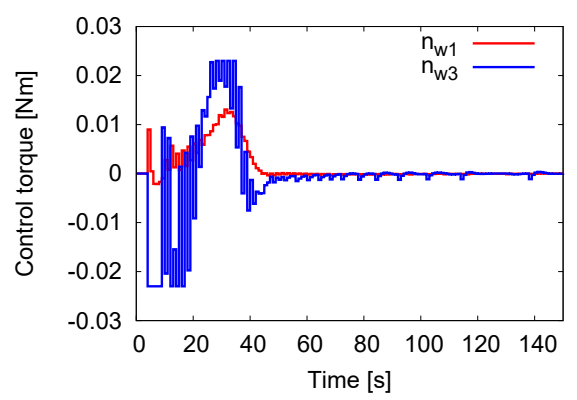

(c)

Fig. 9. Results for SMC for Case 2: (a) angular velocities, (b) trajectory of the target on the screen, and (c) control torques.

that of SMC, as shown in Fig. 8. Moreover, since the finally estimated time delay for ATDESMC (2) was not so different from that for ATDESMC (1), their trajectories were approximately the same. However, a small difference was observed, that is, the trajectory for ATDESMC (1) was slightly more efficiently stabilized to the origin than that of ATDESMC (2).

Next, the results for Case 2 are discussed. As shown in Figs. 9(a) and 9(b), angular velocities converged to zero and the attitude was successfully controlled to stabilize the target at the origin of the camera screen by SMC. Since the element $(1,1)$ of $S$ was set to a smaller value than that for Case 1 (i.e., the relative control gain for $n_{w 1}$ was set to be larger), the value of $p_{1}$ (or $v$ ) was stabilized to zero faster. Due to the faster stabilization of $p_{1}$ (or $v$ ), compared to Case 1 , the trajectory of the target on the camera screen appeared as a straighter line to the origin before $u$ converged to approximately zero. This convergence of $p_{1}$ (or $v$ ) was undesirable for maintaining the controllability of $p_{2}$. An unpredicted small torque was generated when the magnitude of $p_{1}$ became too small, but $p_{2}$ diverged from zero. Similarly to the results for Case 1, ATDESMC succeeded in stabilizing the angular velocities to zero and moving the target to the origin

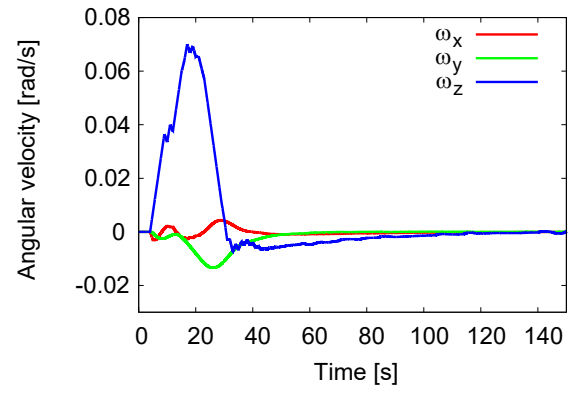

(a)

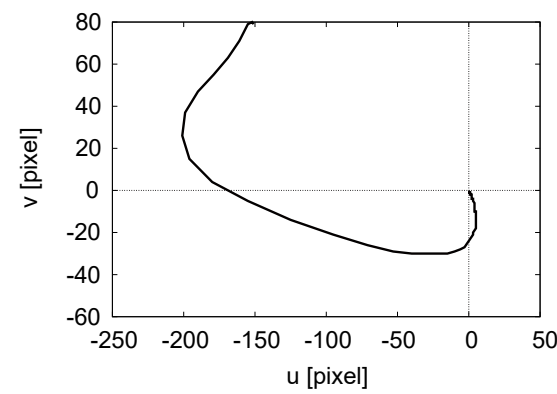

(b)

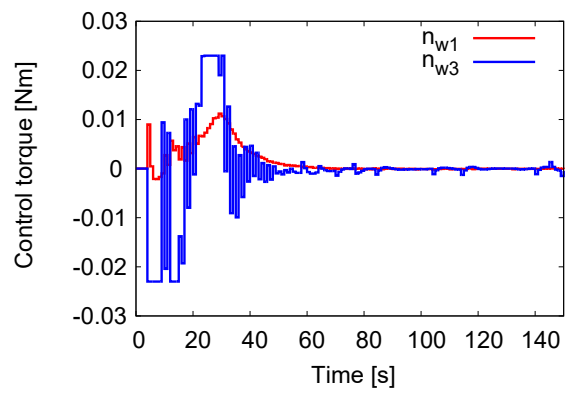

(c)

Fig. 10. Results for ATDESMC for Case 2: (a) angular velocities, (b) trajectory of the target on the screen, (c) control torques.

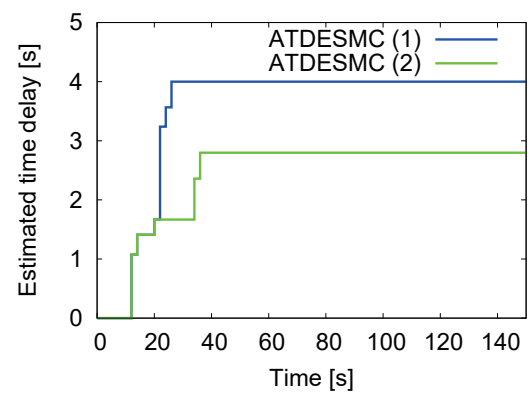

Fig. 11. Time histories of the time-delay estimation for Case 2.

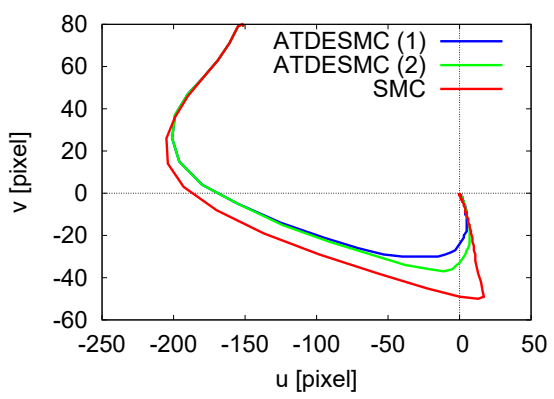

Fig. 12. Comparison of trajectories for Case 2. 
of the camera screen, as shown in Figs. 10(a) and 10(b).

The settling time for ATDESMC was approximately $130 \mathrm{~s}$, the same as that for SMC. Limit cycles were observed in the time history of the control torque for SMC, but their magnitude was much smaller than that in Ref. 30). These limit cycles were a side effect of making the element $(1,1)$ of $\boldsymbol{S}$ smaller. Figure 11 shows the time histories of the estimated time delay for the two values of $\delta$. The estimated time delay was updated until $28 \mathrm{~s}$ for $\operatorname{ATDESMC}(1)$, which approximately corresponded to the timing of the dramatic decrease in angular velocities and change in control torque about the $z$-axis. The finally estimated time delay was approximately $4 \mathrm{~s}$ for ATDESMC. Since the maximum time delay was $4 \mathrm{~s}$, as mentioned earlier, the adaptive time-delay estimation law presented here appropriately estimated the time delay if a sufficiently small value was set for $\delta$. As shown in Fig. 12, the trajectories on the camera screen for the two examples of ATDESMC were shorter than those for SMC. In addition, as shown in Fig. 12, the trajectory for $\delta=2.0 \times 10^{-5}$ (ATDESMC (2)) was slightly longer than that for $\delta=1.0 \times 10^{-7}$ (ATDESMC (1)). This is because, as the value of $\delta$ was set to be larger, the dead band for updating the estimated time delay became larger. As a result, the finally estimated time delay for $\delta=2.0 \times 10^{-5}$ (ATDESMC (2)) was not as accurate as that for $\delta=1.0 \times 10^{-7}(\operatorname{ATDESMC}(1))$. However, as a result of time delay estimation, the trajectory on the camera screen for ATDESMC (2) was still shorter than that of SMC.

The proposed ATDESMC can estimate a time delay that is unknown in advance and is effective for improving the performance of the sliding mode controller for a bias momentum satellite with two wheels when time delay exists in the system.

\section{Conclusions}

This paper proposed an adaptive time-delay estimated sliding mode control (ATDESMC) that uses two wheels to stabilize the attitude of a bias momentum satellite with a time delay. The Rodrigues parameter represents the attitude, and an adaptive law is designed based on the discrete Newton technique so as to detect the time delay in the system by sensing the difference between a predicted attitude and the measured one, and compensating for the time delay by predicting the current state. The results of numerical simulations demonstrated that the proposed adaptive time-delay estimation law can improve the performance of the sliding mode controller without adaptive laws for a bias momentum satellite with two wheels.

\section{References}

1) Crouch, P. E.: Spacecraft Attitude Control and Stabilization: Application of Geometric Control Theory to Rigid Body Model, IEEE Trans. Automatic Control, 29 (1984), pp. 321-331.

2) Brockett, R. W.: Asymptotic Stability and Feedback Stabilization, Differential Geometric Control Theory, 27 (1983), pp. 181-191.

3) Aeyels, D.: Stabilization by Smooth Feedback Control of the Angular Velocity of a Rigid Body, Syst. Control Lett., 6 (1985), pp. 59-63.
4) Aeyels, D. and Szafransski, M.: Comments on the Stabilizability of the Angular Velocity of a Rigid Body, Syst. Control Lett., 10 (1988), pp. 35-39.

5) Sontag, E. D. and Sussman, H. J.: Further Comments on the Stabilizability of the Angular Velocity of a Rigid Body, Syst. Control Lett., 12 (1988), pp. 213-217.

6) Outbib, R. and Sallet, G.: Stabilizability of the Angular Velocity of a Rigid Body Revisited, Syst. Control Lett., 18 (1992), pp. 93-98.

7) Astolfi, A. and Rapaport, A.: Robust Stabilization of the Angular Velocity of a Rigid Body, Syst. Control Lett., 34 (1998), pp. 257-264.

8) Astolfi, A.: Output Feedback Stabilization of the Angular Velocity of a Rigid Body, Syst. Control Lett., 36 (1999), pp. 181-192.

9) Mazenc, F. and Astolfi, A.: Robust Output Feedback Stabilization of the Angular Velocity of a Rigid Body, Syst. Control Lett., 39 (2000), pp. 203-210.

10) Kojima, H.: Stabilization of Angular Velocity of Asymmetrical Rigid Spacecraft Using Two Constant Torques, J. Guid. Control Dynam., 30 (2007), pp. 1163-1168.

11) Krishman, H., Reyhanoglu, M., and McClamroch, H.: Attitude Stabilization of a Rigid Spacecraft Using Two Control Torques: A Nonlinear Control Approach Based on the Spacecraft Attitude Dynamics, Automatica, 30 (1994), pp. 1023-1027.

12) Yoshikawa, S. and Yamada, K.: Motion Control of Tumbling Spacecraft by Repetitive Impulse Inputs, J. Space Technol. Sci., 18 (2002), pp. 17-24.

13) Morin, P., Samson, C., Pomet, J. B., and Jiang, Z. P.: Time-Varying Feedback Stabilization of the Attitude of a Rigid Spacecraft with Two Controls, Syst. Control Lett., 25 (1995), pp. 375-385.

14) Morin, P. and Samson, C.: Time-Varying Exponential Stabilization of a Rigid Spacecraft with Two Control Torques, IEEE Trans. Automatic Control, 42 (1997), pp. 528-533.

15) Coron, J. M. and Kerai, E. Y.: Explicit Feedbacks Stabilizing the Attitude of a Rigid Spacecraft with Two Control Torques, Automatica, 32 (1996), pp. 669-677.

16) Tsiotras, P., Corless, M., and Longuski, M.: A Novel Approach to the Attitude Control of Axisymmetric Spacecraft, Automatica, 31 (1995), pp. 1099-1112.

17) Tsiotras, P.: Optimal Regulation and Passivity Results for Axisymmetric Rigid Bodies Using Two Controls, J. Guid. Control Dynam., 20 (1997), pp. 457-464.

18) Tsiotras, P. and Longuski, J. M.: Spin-Axis Stabilization of Symmetric Spacecraft with Two Control Torques, Syst. Control Lett., 23 (1994), pp. 395-402.

19) Zavoli, A., Matteis, G. D., Giulietti, F., and Avanzini, G.: Single-Axis Pointing of an Underactuated Spacecraft Equipped with Two Reaction Wheels, J. Guid. Control Dynam., 40 (2017), pp. 1465-1472.

20) Shen, H. and Tsiotras, P.: Time-Optimal Control of Axisymmetric Rigid Spacecraft Using Two Controls, J. Guid. Control Dynam., 22 (1999), pp. 682-694.

21) Endo, T. and Ueno, S.: Minimum Energy Maneuver of Satellites by Two Wheels, J. Jpn. Soc. Aeronaut. Space Sci., 51 (2003), pp. 613-620 (in Japanese).

22) Tsiotras, P. and Longuski, J. M.: A New Parameterization of the Attitude Kinematics, J. Astronaut. Sci., 43 (1995), pp. 243-262.

23) Tsuchiya, K., Urakubo, T., Nagaoka, K., and Tsujita, K.: Attitude Control of a Spacecraft with Two Reaction Wheels, Proceedings of 9th Workshop on Astrodynamics and Flight Mechanics, ISAS, 1999, pp. 318-325.

24) Shimoda, S., Kubota, T., and Nakatani, I.: Attitude Control of Satellite with Trajectory Planning using Two Wheels, Proceedings of 12th Workshop on Astrodynamics and Flight Mechanics, ISAS, 2002, pp. 204-209.

25) Shimoda, S., Kubota, T., and Nakatani, I.: Attitude Control of Two Wheel Satellite Considering Maneuver Path, Trans. Soc. Instrument Control Eng., 41 (2005), pp. 813-820 (in Japanese).

26) Yamada, K. and Yoshikawa, S.: Realization of Pure Spin Motion of Spacecraft by Two Wheels, Proceedings of 11th Workshop on Astrodynamics and Flight Mechanics, ISAS, 2001, pp. 77-84.

27) Yamada, K. and Yoshikawa, S.: On Attitude Expression in Pointing Control, Proceedings of 12th Workshop on Astrodynamics and Flight Mechanics, ISAS, 2002, pp. 214-221. 
Trans. Japan Soc. Aero. Space Sci., Vol. 62, No. 4, 2019

28) Kasai, S., Kojima, H., and Satoh, M.: Spacecraft Attitude Maneuver using Two Single-gimbal Control Moment Gyros, Acta Astronautica, 84 (2013), pp. 88-98.

29) Kojima, H., Hiraiwa, K., and Yoshimura, Y.: Experimental Study on Line-of-Sight (LOS) Attitude Control Using Control Moment Gyros under Micro-Gravity Environment, Acta Astronautica, 143 (2018), pp. 118-125.

30) Terui, F., Nagai, Y., Yamamoto, H., Yoshihara, K., Yamamoto, T., and Nakasuka, S.: Moon Tracking Attitude Control Experiment of a
Bias Momentum Micro Satellite $\mu$-LabSat, Trans. Jpn. Soc. Aeronaut. Space Sci., 48 (2005), pp. 28-33.

31) Zheng, G., Polyakov, A., and Levant, A.: Delay Estimation via Sliding Mode for Nonlinear Time-delay Systems, Automatica, 89 (2018), pp. 266-273.

Katsuhiko Yamada Associate Editor 